\title{
GENERALIZED MEAN OF ORDER $t$ VIA BOX AND COX'S TRANSFORMATION
}

\author{
VICENTE QUESADA AND INDER JEET TANEJA
}

\begin{abstract}
Mean of order $t$ and Box and Cox's transformation function are very famous in the literature of mathematics and statistics respectively. In this paper, we have derived some standard inequalities from the mean of order $t$ and studied some interesting properties of Box and Cox's transformation function. A composite relation of these two measures, calling generalized mean of order $t$ or unified $(t, s)$-mean is considered. The unified $(t, s)$-mean leads us to very important generalized information theoretic measures. These measures include generalizations of Shannon's entropy, Kullback-leibler's relative information, Kerridge's inaccura.cy, J-divergence, Jensen difference divergence measure, etc. Properties of unified $(t, s)$-mean are also studied.
\end{abstract}

\section{Mean of Order $t$}

Let $\Delta_{n}=\left\{V=\left(v_{1}, v_{2}, \cdots, v_{n}\right) \mid v_{i} \geq 0, \sum_{i=1}^{n} v_{i}=1\right\}, n \geq 2$ be a set of all complete finite discrete probability distribution. Let $\Gamma_{n}=\left\{W=\left(w_{1}, \cdots, w_{n}\right) \mid w_{i} \geq 0, i=\right.$ $1,2, \cdots, n\} \subset \mathbb{R}^{n}$. For $(V, W) \in \Delta_{n} \times \Gamma_{n}$, the mean of order $t$ is given by

$$
M_{t}(V \| W)= \begin{cases}{\left[\sum_{i=1}^{n} v_{i} w_{i}^{t}\right]^{1 / t},} & t \neq 0 \\ \prod_{i=1}^{n} w_{i}^{v_{i}}, & t=0\end{cases}
$$

where $v_{i}, w_{i}>0$ for $t<0, \forall i$.

In particular, the mean of order $-1,1$ and 2 are the harmonic mean, the arithmetic mean and the root-mean-square respectively. The following result is well known in the literature (ref. Beckenbach and Bellman, 1971, pp.17).

Received October 27, 1992; revised January 24, 1994.

1991 Mathematics Subject Classification. 94A15, 62B10.

Key words and phrases, Mean of order $t$, Box and Cox's transformation, probability distribution, information-theoretic measures, unified $(t, s)$-mean. 
Result 1. We have

(i) $M_{\hat{t}}(V \| W)$ is an increasing function of $t$.

(ii) $t \log M_{\dot{\varepsilon}}(V \| W)$ is convex function of $t$.

(iii) $M_{t}(V \| W)= \begin{cases}w_{\min }, & t \rightarrow-\infty \\ w_{\max }, & t \rightarrow \infty\end{cases}$

where $w_{\min }=\min \left\{w_{1}, \cdots, w_{n}\right\}$ and $w_{\max }=\max \left\{w_{1}, \cdots, w_{n}\right\}$.

(iv) $M_{t}(V \| W) \leq \sum_{i=1}^{n} v_{i} w_{i} \leq M_{1 / t}(V \| W), t \leq 1, t \neq 0$.

(v) $M_{t}(V \| W) \geq \sum_{i=1}^{n} v_{i} w_{i} \geq M_{1 / t}(V \| W), t \geq 1$

\section{Classical inequalities}

In this section, we shall prove some classical inequalities frequentlity used in the literature on mathematics, statistics, information theory etc.. These inequalities are proved as a consequences of the result 1 .

Result 2.(Jensen's inequalities). We have

$$
\sum_{i=1}^{n} v_{i} w_{i}^{t}\left\{\begin{array}{l}
\leq\left[\sum_{i=1}^{n} v_{i} w_{i}\right]^{t}, \quad 0<t<1 \\
\geq\left[\sum_{i=1}^{n} v_{i} w_{i}\right]^{t}, \quad t>1 \text { or } t<0
\end{array}\right.
$$

with equality iff $w_{i}=c, \forall i=1,2, \cdots, n$.

Proof. From the parts (iv) and (v) of result 1 , we have

$$
\left[\sum_{i=1}^{n} u_{i}^{t} v_{i}^{t}\right]^{1 / t}\left\{\begin{array}{l}
\leq \sum_{i=1}^{n} v_{i} w_{i}, \quad t<1, t \neq 0 \\
\geq \sum_{i=1}^{n} v_{i} w_{i}, \quad t>1
\end{array}\right.
$$

with equality iff $w_{i}=c, \forall i=1,2, \cdots, n$.

Raising both side of the inequalities (3) by $t$, we get the required result.

In the inequalities (2) take $w_{i}=u_{i} / v_{i}, \forall i$, we get

$$
\left[\sum_{i=1}^{n} u_{i}^{t} v_{i}^{1-t}\right]^{1 / t}\left\{\begin{array}{l}
\leq \sum_{i=1}^{n} u_{i}, \quad t<1, t \neq 0 \\
\geq \sum_{i=1}^{n} u_{i}, \quad t>1
\end{array}\right.
$$

i.e.,

$$
\sum_{i=1}^{n} u_{i}^{t} v_{i}^{1-t} \begin{cases}\leq\left[\sum_{i=1}^{n} u_{i}\right]^{t}, & 0<t<1 \\ \geq\left[\sum_{i=1}^{n} u_{i}\right]^{t}, & t>1 \text { or } t<0\end{cases}
$$


with equality iff $u_{i}=c v_{i}, \forall i$, i.e., iff $v_{i}=u_{i} / \sum_{i=1}^{n} u_{i}, \forall i$.

In the inequalities (4) take $u_{i}=p_{i}$, we get

$$
\sum_{i=1}^{n} p_{i}^{t} v_{i}^{1-t}\left\{\begin{array}{l}
\leq 1, \quad 0<t<1 \\
\geq 1, \quad t>1 \text { or } t<0
\end{array}\right.
$$

with equality iff $p_{i}=v_{i}, \forall i$, where $P=\left(p_{1}, p_{2}, \cdots, p_{n}\right) \in \triangle_{n}$. For $t<0, p_{i}>0$ and $v_{i}>0, \forall i$.

Thus the inequalities (4) and (5) are the consequences of the Jensen's inequalities. While the inequalities (3) can be considered equivalent version of the inequalities (2).

Result 3. (Hölder's inequalities). We have

$$
\left[\sum_{i=1}^{n} a_{i}^{p}\right]^{\frac{1}{p}}\left[\sum_{i=1}^{n} b_{i}^{q}\right]^{\frac{1}{q}} \begin{cases}\leq \sum_{i=1}^{n} a_{i} b_{i}, & p<1, p \neq 0 \\ \geq \sum_{i=1}^{n} a_{i} b_{i}, & p>1\end{cases}
$$

with equality iff for some $c, a_{i}^{p}=c b_{i}^{q}, \forall i$, where $a_{i}$, and $b_{i}$ are nonnegative real numbers and $\frac{1}{p}+\frac{1}{q}=1$. For $p<0, a_{i}>0, b_{i}>0, \forall i$.

Proof. Take $v_{i}=b_{i}^{t /(t-1)} / \sum_{i=1}^{n} b_{i}^{t /(t-1)}$ and $w_{i}=a_{i} b_{i}^{1 /(1-t)} \sum_{i=1}^{n} b_{i}^{t /(t-1)}, t \neq 1$ in the inequalities (3), we get

$$
\left[\sum_{i=1}^{n} \frac{b_{i}^{t /(i-1)}}{\sum_{i=1}^{n} b_{i}^{t /(t-1)}} a_{i}^{t} b_{i}^{t /(1-t)}\left[\sum_{i=1}^{n} b_{i}^{t /(t-1)}\right]^{t}\right]^{1 / t} \begin{cases}\leq \sum_{i=1}^{n} a_{i} b_{i}, & t<1, t \neq 0 \\ \geq \sum_{i=1}^{n} a_{i} b_{i}, & t>1\end{cases}
$$

i.e.,

$$
\left[\sum_{i=1}^{n} a_{i}^{t}\right]^{\frac{1}{2}}\left[\sum_{i=1}^{n} b_{i}^{t /(t-1)}\right]^{\frac{t-1}{i}} \begin{cases}\leq \sum_{i=1}^{n} a_{i} b_{i}, & t<1, t \neq 0 \\ \geq \sum_{i=1}^{n} a_{i} b_{i}, & t>1\end{cases}
$$

with equality iff $a_{i} b_{i}^{1 /(1-t)} \sum_{i=1}^{n} b_{i}^{t /(t-1)}=c$, i.e., iff $a_{i}^{t}=c^{\prime} b_{i}^{t /(t-1)} \forall i$.

The above expression is same as (6) for $t=p$ and $q=\frac{t}{t-1}$

The inequalities (6) can also be derived from (4) by taking $u_{i}=a_{i} b_{i}$ and $v_{i}=$ $b_{i}^{t(t-1)} / \sum_{i=1}^{n} b_{i}^{t /(t-1)}, t \neq 1, \forall i$.

The equivalent version of the inequalities (6) is given by

$$
\left[\sum_{i=1}^{n} a_{i}^{\frac{1}{l}}\right]^{t}\left[\sum_{i=1}^{n} b_{i}^{\frac{1}{1-t}}\right]^{1-t}\left\{\begin{array}{l}
\geq \sum_{i=1}^{n} a_{i} b_{i}, \quad 0<t<1, \\
\leq \sum_{i=1}^{n} a_{i} b_{i}, \quad t>1 \text { or } t<0
\end{array}\right.
$$


with equality iff $a_{i}^{t}=c b_{i}^{1-t}, \forall i$, where $a_{i}$, and $b_{i}$ are nonnegative real numbers. For $t<0, a_{i}>0, b_{i}>0, \forall i$.

The proof of the inequalities (7) follows from (6) by taking $p=\frac{1}{t}$.

Result 4. (Generalized Holder's inequalities). We have

$$
\left[\sum_{i=1}^{n} a_{i}^{p} g_{i}\right]^{\frac{1}{p}}\left[\sum_{i=1}^{n} b_{i}^{q} g_{i}\right]^{\frac{1}{q}} \begin{cases}\leq \sum_{i=1}^{n} a_{i} b_{i} g_{i}, & p<1, p \neq 0 \\ \geq \sum_{i=1}^{n} a_{i} b_{i} g_{i}, & p>1\end{cases}
$$

with equality iff for some $c, g_{i} a_{i}^{p}=c g_{i} b_{i}^{q}, \forall i$, where $a_{i}, b_{i}$ and $g_{i}$ are nonnegative real numbers and $\frac{1}{p}+\frac{1}{q}=1$. For $p<0, a_{i}>0, b_{i}>0, \forall i$.

Proof. Take $a_{i}=\left(s_{i} g_{i}\right)^{t}$ and $b_{i}=\left(k_{i} g_{i}\right)^{1-t}$ in $(7)$, we get

$$
\left[\sum_{i=1}^{n} s_{i} g_{i}\right]^{t}\left[\sum_{i=1}^{n} k_{i} g_{i}\right]^{1-t} \begin{cases}\geq \sum_{i=1}^{n} s_{i}^{t} k_{i}^{1-t} g_{i}, & 0<t<1 \\ \leq \sum_{i=1}^{n} s_{i}^{t} k_{i}^{1-t} g_{i}, & t>1 \text { ort }<0\end{cases}
$$

By taking $s_{i}=a_{i}^{p}, k_{i}=b_{i}^{q}$ and $t=\frac{1}{p}$ in (9), we get (8).

The equivalent version of the inequalities (8) is given by

$$
\left[\sum_{i=1}^{n} a_{i}^{1 / t} g_{i}\right]^{t}\left[\sum_{i=1}^{n} b_{i}^{1(1-t)} g_{i}\right]^{1-t}\left\{\begin{array}{l}
\geq \sum_{i=1}^{n} a_{i} b_{i} g_{i}, \quad 0<t<1, \\
\leq \sum_{i=1}^{n} a_{i} b_{i} g_{i}, \quad t>1 \text { or } t<0
\end{array}\right.
$$

with equality iff for some $c, g_{i} a_{i}^{1 / t}=c g_{i} b_{i}^{1 /(1-t)}, \forall i$, where $a_{i}, b_{i}$ and $g_{i}$ are nonnegative real numbers. For $t<0, a_{i}>0, b_{i}>0, g_{i}>0 \forall i$.

the proof follows by taking $s_{i}=a_{i}^{1 / t}$ and $k_{i}=b_{i}^{1 /(1-t)}$ in (9).

Result 5. (Minköwski's inequalities). We have

$$
\sum_{k=1}^{n}\left[\sum_{j=1}^{n} a_{j k}^{t}\right]^{1 / t} \begin{cases}\leq\left[\sum_{j=1}^{n}\left[\sum_{k=1}^{n} a_{j k}\right]^{t}\right]^{1 / t}, & t<1, t \neq 0 \\ \geq\left[\sum_{j=1}^{n}\left[\sum_{k=1}^{n} a_{j k}\right]^{t}\right]^{1 / t}, & t>1\end{cases}
$$

with equality iff $a_{j k}$ are independent of $j$, i.e., $a_{j k}=c_{k}, \forall j, k$, where $a_{j k}$ are nonnegative real numbers. For $t<0, a_{j k}>0 \forall j, k$. 
Proof. In the inequalities (6) take $a_{j}=a_{j k}, b_{j}=\left[\sum_{i=1}^{n} a j i\right]^{t-1}$ and $p=t$, we get

$$
\left[\sum_{j=1}^{n} a_{j k}^{t}\right]^{1 / t}\left[\sum_{j=1}^{n}\left[\sum_{i=1}^{n} a_{j i}\right]^{t}\right]^{(t-1) / t}\left\{\begin{array}{l}
\leq \sum_{j=1}^{n} a_{j k}\left[\sum_{i=1}^{n} a_{j i}\right]^{t-1}, t<1, t \neq 0 \\
\geq \sum_{j=1}^{n} a_{j k}\left[\sum_{i=1}^{n} a_{j i}\right]^{t-1}, t>1
\end{array}\right.
$$

Summing both sides of the above inequality over all $k$, and simplifying,we get

$$
\sum_{k=1}^{n}\left[\sum_{j=1}^{n} a_{j k}^{t}\right]^{1 / t}\left[\sum_{j=1}^{n}\left[\sum_{i=1}^{n} a_{j i}\right]^{t}\right]^{(t-1) / t} \begin{cases}\leq \sum_{j=1}^{n}\left[\sum_{k=1}^{n} a_{j k}\right]^{t}, & t<1, t \neq 0 \\ \geq \sum_{j=1}^{n}\left[\sum_{k=1}^{n} a_{j k}\right]^{t}, & t>1\end{cases}
$$

with equality iff $a_{j k}^{t}=c\left[\sum_{k=1}^{n} a_{j k}\right]^{t}$, i.e., iff $a_{j k}=c^{\prime} \sum_{k=1}^{n} a_{j k}$, i.e., iff $a_{j k}$ are independent of $k$, where

$$
\sum_{k=1}^{n}\left[\sum_{j=1}^{n} a_{j k}\right]\left[\sum_{i=1}^{n} a_{j i}\right]^{t-1}=\sum_{j=1}^{n}\left[\sum_{k=1}^{n} a_{j k}\right]\left[\sum_{i=1}^{n} a_{j i}\right]^{t-1}=\sum_{j=1}^{n}\left[\sum_{k=1}^{n} a_{j k}\right]^{t} .
$$

Simplifying the expression (12), we get the required result.

The equivalent version of the inequalities (11) is given by

$$
\sum_{k=1}^{n}\left[\sum_{j=1}^{n} a_{j k}^{1 / t}\right]^{t}\left\{\begin{array}{l}
\geq\left[\sum_{j=1}^{n}\left[\sum_{k=1}^{n} a_{j k}\right]^{1 / t}\right]^{t}, \quad 0<t<1 \\
\leq\left[\sum_{j=1}^{n}\left[\sum_{k=1}^{n} a_{j k}\right]^{1 / t}\right]^{t}, \quad t>1 \text { or } t<0
\end{array}\right.
$$

with equality iff $a_{j k}$ are independent of $j$, i.e., $a_{j k}=c_{k}, \forall j, k$, where $a_{j k}$ are nonnegative real numbers. For $t<0, a_{j k}>0 \forall j, k$.

The proof of the inequalities (13) follows from (7) by taking $a_{j}=a_{j k}$ and $b_{j}=\left[\sum_{i=1}^{n} a_{j i}\right]^{1-t}$.

Result 6. (Generalized Minköwski's inequalities). We have

$$
\sum_{k=1}^{n}\left[\sum_{j=1}^{n} g_{j} a_{j k}^{t}\right]^{1 / t}\left\{\begin{array}{l}
\leq\left[\sum_{j=1}^{n}\left[\sum_{k=1}^{n} a_{j k}\right]^{t} g_{j}\right]^{1 / t}, \quad t<1, t \neq 0 \\
\geq\left[\sum_{j=1}^{n}\left[\sum_{k=1}^{n} a_{j k}\right]^{t} g_{j}\right]^{1 / t}, t>1
\end{array}\right.
$$


where $a_{j k}$ and $g_{j}$ are nonnegative real numbers. For $t<0, a_{j k}>0, g_{j}>0 \forall j, k$.

Proof. Take $a_{j k}=g_{j}^{1 / t} a_{j k}$ in the inequalities (11), we get (14). The equivalent version of the inequalities (14) is given by

$$
\sum_{k=1}^{n}\left[\sum_{j=1}^{n} a_{j k}^{1 / t} g_{j}\right]^{t}\left\{\begin{array}{l}
\geq\left[\sum_{j=1}^{n}\left[\sum_{k=1}^{n} a_{j} k\right]^{1 / t} g_{j}\right]^{t}, \quad 0<t<1 \\
\leq\left[\sum_{j=1}^{n}\left[\sum_{k=1}^{n} a_{j k}\right]^{1 / t} g_{j}\right]^{t}, \quad t>1 \text { or } t<0
\end{array}\right.
$$

The inequalities (15) follows from (13) by taking $a_{j k}=a_{j k} g_{j}^{t}$.

Remark 1. Gallager (1968, pp 522-524) and Jelinek (1968, pp. 510-518) gave very importance to the above inequalites and apply them to prove some important results on information theory. Jelinek considered these inequalities for convex numbers and extended some of these for integral forms. Gallager considered only for real numbers. The parameters considered by both of them are for positive values, while we have extended them for the negative values too. The equivalent version of the inequalities given are also sometimes useful in applications. Some of these inequalities can be seen in Backenbach and Bellman (1971).

\section{Box And Cox's Transformation Function}

Let us consider the following function

$$
\theta_{s}(x)= \begin{cases}\left(2^{1-s}-1\right)^{-1}\left[x^{s-1}-1\right], & s \neq 1 \\ -\log _{2} x, & s=1\end{cases}
$$

for all $s \in(-\infty, \infty)$ and $x \in(0, \infty)$.

The function $\theta_{s}(x)$ is famous as Box and Cox's transformation (Box and Cox, 1964) and found its applictions in Statistics (Box and Tiao, 1973). For more details refer to Broemeling (1982). Some interesting properties are given in the following result.

Result 7. We have

(i) $\theta_{s}(x) \begin{cases}\geq 0, & 0 \leq x \leq 1 \\ \leq 0, & x \geq 1\end{cases}$

with equality iff $\bar{x}=1$.

(ii) $\lim _{x \rightarrow 0^{+}} \theta_{s}(x)= \begin{cases}\infty, & s \leq 1 \\ \left(1-2^{1-s}\right)^{-1}, & s>1\end{cases}$

(iii) $\lim _{x \rightarrow \infty} \theta_{s}(x)= \begin{cases}1, & 0<x \leq 1 \\ 0, & x=1 \\ -\infty, & x>1\end{cases}$ 
(iv) $\lim _{s \rightarrow-\infty} \theta_{s}(x)= \begin{cases}\infty, & 0<x<\frac{1}{2} \\ 1, & x=\frac{1}{2} \\ 0, & x>\frac{1}{2}\end{cases}$

(v) $\theta_{s}(x)$ is a convex function in $x$ for $s<2$.

(vi) $\theta_{s}(x)$ is a concave function in $x$ for $s>2$.

(vii) $\theta_{s}(x)$ is a monotonically decreasing function of $x$.

(viii) $\theta_{s}(x y)=\theta_{s}(x)+\theta_{s}(y)+\left(2^{1-s}-1\right) \theta_{s}(x) \theta_{s}(y)$, for all $x, y \in \mathbb{R}^{+}$.

(ix) $\theta_{s}(x)$ is a monotonoically decreasing function of $s$ for $x \geq 1$.

(x) $\theta_{s}(x) \begin{cases}\leq k(s) \theta_{1}(x), & s \geq 1 \\ \geq k(s) \theta_{1}(x), & s \leq 1\end{cases}$

where

$$
\theta_{1}(x)=\lim _{s \rightarrow 1} \theta_{s}(x)=-\log _{2} x
$$

and

$$
k(s)= \begin{cases}\left(2^{1-s}-1\right)^{-1}(1-s) \ln 2, & s \neq 1 \\ 1, & s=1\end{cases}
$$

with

$k(s) \begin{cases}\geq 1, & s \geq 1 \\ \leq 1, & s \leq 1\end{cases}$

(xi) $\theta_{s}(x)= \begin{cases}\geq \theta_{1}(x),\left(\theta_{1}(x) \geq 1, s \leq 1\right) & \text { or } \quad\left(0 \leq \theta_{1}(x) \leq 1, s \geq 1\right) \\ \leq \theta_{1}(x),\left(0 \leq \theta_{1}(x) \leq 1, s \leq 1\right) & \text { or } \quad\left(\theta_{1}(x) \geq 1, s \geq 1\right)\end{cases}$

with equality iff $x=1$ or $s=1$.

(xii) $1-x\left\{\begin{array}{l}\leq \frac{1}{2} \theta_{s}(x),\left(0<x \leq \frac{1}{2} \text { or } x \geq 1, s \leq 2\right) \text { or }\left(\frac{1}{2} \leq x \leq 1, s \geq 2\right) \\ \geq \frac{1}{2} \theta_{s}(x),\left(\frac{1}{2} \leq x \leq 1, s \leq 2\right) \text { or }\left(0<x \leq \frac{1}{2} \text { or } x \geq 1, s \geq 2\right)\end{array}\right.$

with equality iff $x=\frac{1}{2}$ or $x=1$ or $s=2$.

Proof. parts (i) to (viii) are easy verifications.

(ix) For $s \neq 1$, we have

$$
\begin{aligned}
\frac{d}{d s}\left(\theta_{s}(x)\right) & =(-1)\left(2^{1-s}-1\right)^{-2} 2\left(\frac{1}{2}\right)^{s}\left(\ln \frac{1}{2}\right)\left(x^{s-1}-1\right)+\left(2^{1-s}-1\right)^{-1}\left(x^{-1} x^{s} \ln x\right) \\
& =(-1)\left(2^{1-s}-1\right)^{-2}\left[\frac{x^{s-1}-1}{s-1} 2^{1-s} \ln 2^{1-s}+\frac{1-2^{1-s}}{s-1} x^{s-1} \ln x^{s-1}\right]
\end{aligned}
$$

Since

$$
a \ell \mathrm{n} a \geq a-1, a \geq 0 \quad(0 \log 0=0)
$$

and

$$
\frac{x^{s-1}-1}{s-1} \begin{cases}\geq 0, & x \geq 1 \\ \leq 0, & 0<x \leq 1\end{cases}
$$

hold, then we conclude that

$$
\frac{d}{d s}\left(\theta_{s}(x)\right) \leq 0 \text { for } x \geq 1
$$

This proves that the function $\theta_{s}(x)$ is monotonically decreasing in $s$ for $x \geq 1$. 
(x) We know that

$$
\ln a \leq a-1, a>0 \text {. }
$$

Taking $a=x^{s-1}, x>0$ and $a=2^{1-s}$, and rearranging the terms, we get the required result.

(xi) For $a>0$, we know that (ref. Hardy et al., 1934, pp.40, Th. 4.2)

$$
a^{\gamma}-1 \begin{cases}\geq \gamma(a-1), & \gamma \geq 1 \\ \leq \gamma(a-1), & 0<\gamma \leq 1\end{cases}
$$

Take $a=2^{1-s}$ and $\gamma=-\log _{2} x$, we get the result.

(xii) Let us consider the function

$$
\eta_{s}(x)=1-x-\frac{1}{2} \theta_{s}(x) .
$$

Then

$$
\eta_{s}^{\prime}(x)=-1-\frac{1}{2} \theta_{s}^{\prime}(x)
$$

and

$$
\eta_{s}^{\prime \prime}(x)=-\frac{1}{2} \theta_{s}^{\prime \prime}(x)
$$

where

$$
\theta_{s}^{\prime}(x)= \begin{cases}\left(2^{1-s}-1\right)^{-1}(s-1) x^{s-2}, & s \neq 1 \\ -\frac{1}{\ln 2} \frac{1}{x}, & s=1\end{cases}
$$

and

$$
\theta_{s}^{\prime \prime}(x)= \begin{cases}\left(2^{1-s}-1\right)^{-1}(s-1)(s-2) x^{s-3}, & s \neq 1 \\ \frac{1}{\ln 2} \frac{1}{x^{2}}, & s=1\end{cases}
$$

Since $\frac{s-1}{2^{1-s}-1}<0$ for any $s$, then

$$
\theta_{s}^{\prime \prime}(x) \begin{cases}>0, & s<2 \\ =0, & s=2 \\ <0, & s>2\end{cases}
$$

This implies that

$$
\eta_{s}^{\prime \prime}(x)=-\frac{1}{2} \theta_{s}^{\prime \prime}(x) \begin{cases}<0, & s<2 \\ =0, & s=2 \\ >0, & s>2\end{cases}
$$

Thus we conclude that $\eta_{s}(x)$ is strictly concave for $s<2$ and is strictly convex for $s>2$. It will attain its maximum or minimum at $\eta_{s}^{\prime}(x)=0$, i.e., when

$$
x= \begin{cases}{\left[2\left(\frac{2^{1-s}-1}{1-s}\right)\right]^{\frac{1}{s-2}},} & s \neq 1, s \neq 2 \\ \frac{1}{2 \ell_{2} 2}, & s=1\end{cases}
$$


i.e., at this point of $x$, the function $\eta_{s}(x)$ attain its maximum or minimum.

Also, the only zeros of $\eta_{s}(x)$ are when $x=\frac{1}{2}$ or $x=1$ or $s=2$.

Using part (ii), we conclude that

$$
\lim _{x \rightarrow 0^{+}} \eta_{s}(x)= \begin{cases}-\infty, & s \leq 1 \\ \frac{2^{2-s}-1}{2\left(2^{1-s}-1\right)}, & s>1\end{cases}
$$

Since

$$
\frac{2^{2-s}-1}{2\left(2^{1-s}-1\right)} \begin{cases}<0, & 1<s<2 \\ =0, & s=2 \\ >0, & s>2\end{cases}
$$

this gives

$$
\lim _{x \rightarrow 0^{+}} \eta_{s}(x) \begin{cases}=-\infty, & s \leq 1 \\ <0, & 1<s<2 \\ =0, & s=2 \\ >0, & s>2\end{cases}
$$

The above expression along with other consideration completes the result.

\section{Generalized Mean of Order $t$ : Unified $(t, s)$-Mean}

Let us consider the following composite relation:

$$
\Phi_{t}^{s}(V \| W)=\theta_{s}\left(M_{t}(V \| W)\right)
$$

for all $(V, W) \in \Delta_{n} \times \Gamma_{n}$. Then

$$
\Phi_{t}^{s}(V \| W)= \begin{cases}\phi_{t}^{s}(V \| W)=\left(2^{1-s}-1\right)^{-1}\left[\left[\sum_{i=1}^{n} v_{i} w_{i}^{t}\right]^{\frac{s-1}{t}}-1\right], & t \neq 0, s \neq 1 \\ \phi_{0}^{s}(V \| W)=\left(2^{1-s}-1\right)^{-1}\left[\left[\prod_{i=1}^{n} w_{i}^{v_{i}}\right]^{s-1}-1\right], & t=0, s \neq 1 \\ \phi_{t}^{1}(V \| W)=-\frac{1}{t} \log _{2}\left[\sum_{i=1}^{n} v_{i} w_{i}^{t}\right], & t \neq 0, s=1 \\ \phi(V \| W)=-\log _{2}\left[\prod_{i=1}^{n} w_{i}^{v_{i}}\right], & t=0, s=1\end{cases}
$$

for all $t, s \in(-\infty, \infty),(V, W) \in \triangle_{n} \times \Gamma_{n}$.

We shall call the measure $\Phi_{t}^{s}(V \| W)$, the unified $(t, s)$-mean or generalized mean of order $t$. It can be written as:

$$
\Phi_{t}^{s}(V \| W)=C E\left\{\phi_{t}^{s}(V \| W) \mid t \neq 0, s \neq 1\right\}
$$

Where "CE" stands for continuous extension. The following results hold: 
Result 8. We have

(i) $\Phi_{t}^{s}(V \| W)$ is a monotonically decreasing function of $t$ ( $s$ fixed).

(ii) $\Phi_{t}^{s}(V \| W)$ is a monotonically decreasing function of $s$ ( $t$ fixed) provided $M_{t}(V \| W) \geq$ 1 .

Proof of part (i) follows from Result 1(i) and of part (ii) follows from Result 7(ix).

Result 9. We have

(i) $\Phi_{i}^{s}(V \| W) \begin{cases}\geq 0, & 0 \leq M_{t}(V \| W) \leq 1 \\ \leq 0, & M_{t}(V \| W) \geq 1\end{cases}$

(ii) $\Phi_{\infty}^{s}(V \| W) \leq \Phi_{t}^{s}(V \| W) \leq \Phi_{-\infty}^{s}(V \| W)$, where

$$
\Phi_{\infty}^{s}(V \| W)=\lim _{t \rightarrow \infty} \Phi_{t}^{s}(V \| W)=\theta_{s}\left(w_{\max }\right)
$$

and

$$
\Phi_{-\infty}^{s}(V \| W)=\lim _{t \rightarrow \infty} \Phi_{t}^{s}(V \| W)=\theta_{s}\left(w_{\min }\right) .
$$

(iii) $\Phi_{i}^{\infty}(V \| W) \leq \Phi_{i}^{s}(V \| W) \leq \Phi_{t}^{-\infty}(V \| W)$,

where

$$
\Phi_{i}^{\infty}(V \| W)=\lim _{s \rightarrow \infty} \Phi_{t}^{s}(V \| W)= \begin{cases}1, & 0<M_{t}(V \| W)<1 \\ 0, & M_{t}(V \| W)=1 \\ -\infty, & M_{t}(V \| W)>1\end{cases}
$$

and

$$
\Phi_{t}^{-\infty}(V \| W)=\lim _{s \rightarrow \infty} \Phi_{t}^{s}(V \| W)= \begin{cases}\infty, & 0<M_{t}(V \| W)<\frac{1}{2} \\ 1, & M_{t}(V \| W)=\frac{1}{2} \\ 0, & M_{t}(V \| W)>\frac{1}{2}\end{cases}
$$

(iv) $\Phi_{t}^{s}(V \| W) \begin{cases}\geq \Phi_{0}^{s}(V \| W), & t \leq 0 \\ \leq \Phi_{0}^{s}(V \| W), & t \geq 0\end{cases}$

(v) $\Phi_{t}^{s}(V \| W) \begin{cases}\geq k(s) \Phi_{t}^{1}(V \| W), & s \leq 1 \\ \leq k(s) \Phi_{t}^{1}(V \| W), & s \geq 1\end{cases}$

where $k(s)$ is as given in (17).

(vi) If $M_{i}(V \| W) \geq 1$ or $\Phi_{t}^{1}(V \| W) \geq 1$, then

$\Phi_{t}^{s}(V \| W) \begin{cases}\geq \Phi_{t}^{1}(V \| W), & s \leq 1 \\ \leq \Phi_{t}^{1}(V \| W), & s \geq 1\end{cases}$

(vii) If $0 \leq \Phi_{t}^{1}(V \| W) \leq 1$, then

$\Phi_{i}^{s}(V \| W) \begin{cases}\geq \Phi_{t}^{1}(V \| W), & s \geq 1 \\ \leq \Phi_{t}^{1}(V \| W), & s \leq 1\end{cases}$

The proof follows as a consequence of the results 1 and 7 .

Result 10. We have

(i) $1-w_{\max } \leq \frac{1}{2} \Phi_{t}^{s}(V \| W)$,

for $\left(0<w_{\max } \leq \frac{1}{2}\right.$ or $\left.\quad w_{\max } \geq 1, s \leq 2\right)$ or $\left(\frac{1}{2} \leq w_{\max } \leq 1, s \geq 2\right)$

(ii) $1-w_{\min } \geq \frac{1}{2} \Phi_{t}^{s}(V \| W)$,

for $\left(0<w_{\min } \leq \frac{1}{2}\right.$ or $\left.w_{\min } \geq 1, s \geq 2\right)$ or $\left.\frac{1}{2} \leq w_{\min } \leq 1, s \leq 2\right)$. 
Proof. Replace $x$ by $w_{\max }$ in Result 7 (xii) and applying the left hand side of Result 9(ii), we get the proof of part (i). Again replacing $x$ by $w_{\min }$ in Result 7(xii) and applying the right hand side of Result 9(ii), we get the proof of part (ii).

Result 11. we have

(i) If $M_{0}\left(V_{1} \| W_{1}\right) \leq M_{0}\left(V_{2} \| W_{2}\right)$, then

$\Phi_{t}^{s}\left(V_{1} \| W_{1}\right) \geq \Phi_{t}^{s}\left(V_{2} \| W_{2}\right)$,

for all $t \in\left(t_{1}, t_{2}\right)$, where $t_{1}$ and $t_{2}$ are determined by the equations

$$
M_{t_{1}}\left(V_{1} \| W_{1}\right)=M_{0}\left(V_{2} \| W_{2}\right)
$$

and

$$
M_{t_{2}}\left(V_{2} \| W_{2}\right)=M_{0}\left(V_{1} \| W_{1}\right) .
$$

(ii) If $M_{0}\left(V_{2} \| W_{2}\right) \geq w_{1 \max }$ and $M_{0}\left(V_{1} \| W_{1}\right) \leq w_{2 \min }$, then

$$
\Phi_{t}^{s}\left(V_{1} \| W_{1}\right) \geq \Phi_{t}^{s}\left(V_{2} \| W_{2}\right)
$$

for all $t, s \in(-\infty, \infty)$.

Proof. (i) We have

$$
\Phi_{0}^{1}\left(\left(V_{1} \| W_{1}\right) \geq \Phi_{0}^{1}\left(V_{2} \| W_{2}\right) \Leftrightarrow M_{0}\left(V_{1} \| W_{1}\right) \leq M_{0}\left(V_{2} \| W_{2}\right) .\right.
$$

In view of (19) and the monotonicity of $M_{t}$ with respect to $t$, there exists $t_{1}<0$ such that

$$
M_{t_{1}}\left(V_{2} \| W_{2}\right)=M_{0}\left(V_{1} \| W_{1}\right)
$$

In a similar manner there exists $t_{2}>0$ such that

$$
M_{t_{2}}\left(V_{1} \| W_{1}\right)=M_{0}\left(V_{2} \| W_{2}\right)
$$

From (20), we have

$$
M_{t}\left(V_{2} \| W_{2}\right) \geq M_{t_{1}}\left(V_{2} \| W_{2}\right)=M_{0}\left(V_{1} \| W_{1}\right) \geq M_{t}\left(V_{1} \| W_{1}\right), \forall t \in\left(t_{1}, 0\right] .
$$

From (21), we have

$$
M_{t}\left(V_{2} \| W_{2}\right) \geq M_{0}\left(V_{2} \| W_{2}\right)=M_{t_{2}}\left(V_{1} \| W_{1}\right) \geq M_{t}\left(V_{1} \| W_{1}\right), \forall t \in\left[0, t_{2}\right) .
$$

From (22) and (23), we conclude that

$$
M_{t}\left(V_{1} \| W_{1}\right) \leq M_{t}\left(V_{2} \| W_{2}\right), \forall t \in\left(t_{1}, t_{2}\right)
$$

The rest part of the proof follows in view of monotonicity of $\theta_{s}(x)$ with respect to $x$ and the continuity of $M_{t}$ with respect to $t$. 
(b) Since $\lim _{t \rightarrow \infty} M_{t}\left(V_{1} \| W_{1}\right)=w_{1 \max }$, then for all $t \in[0, \infty)$, we have

$$
M_{i}\left(V_{2} \| W_{2}\right) \geq M_{0}\left(V_{2} \| W_{2}\right) \geq \lim _{t \rightarrow \infty} M_{t}\left(V_{1} \| W_{1}\right) \geq M_{t}\left(V_{1} \| W_{1}\right)
$$

i.e.,

$$
M_{t}\left(V_{2} \| W_{2}\right) \geq M_{t}\left(V_{1} \| W_{1}\right), \quad 0 \leq t<\infty .
$$

Again, we know that $\lim _{t \rightarrow \infty} M_{t}\left(V_{2} \| W_{2}\right)=w_{2 \min }$, then for all $t \in(-\infty, 0]$, we have

$$
M_{t}\left(V_{2} \| W_{2}\right) \geq M_{-\infty}\left(V_{2} \| W_{2}\right) \geq M_{0}\left(V_{1} \| W_{1}\right) \geq M_{t}\left(V_{1} \| W_{1}\right)
$$

i.e.,

$$
M_{t}\left(V_{2} \| W_{2}\right) \geq M_{t}\left(V_{1} \| W_{1}\right), \quad-\infty<t \leq 0
$$

Combining (24) and (25), we have

$$
M_{t}\left(V_{2} \| W_{2}\right) \geq M_{t}\left(V_{1} \| W_{1}\right), \quad-\infty<t<\infty
$$

Since $\theta_{s}(x)$ is monotonically decreasing in $x$, it gives

$$
\theta_{s}\left(M_{t}\left(V_{2} \| W_{2}\right)\right) \leq \theta_{s}\left(M_{t}\left(V_{1} \| W_{1}\right)\right)
$$

i.e.,

$$
\Phi_{t}^{s}\left(V_{1} \| W_{1}\right) \geq \Phi_{t}^{s}\left(V_{2} \| W_{2}\right)
$$

for all $t, s \in(-\infty, \infty)$.

\section{Information Theoretic Examples of Unified $(t, s)$-Mean}

In this section we shall present some information theoretic examples of the unified $(t, s)$-mean given in section 2 . These examples give unified $(r, s)$-information measures generalizing Shannon's entropy, Kerridge's inaccuracy, Kullback-Leibler's relative information, J-divergence and Jensen difference divergence measure. Some properties are also deduced. It is understood that $r, s \in(-\infty, \infty)$ and $P, Q \in \triangle_{n}^{r}$, where

$$
\Delta_{n}^{r}=\left\{P=\left(p_{1}, \cdots, p_{n}\right) \mid \sum_{i=1}^{n} p_{i}=1 \text { with } p_{i} \geq 0, \text { for } r>0 \text { and } p_{i}>0 \text { for } r \leq 0\right\}
$$

\subsection{Unified $(r, s)$-Entropy}

Take $v_{i}=w_{i}=p_{i}, \forall i=1,2, \cdots, n$ and $t=r-1$ in (18), we get

$$
\Phi_{t}^{s}(V \| W)=\mathcal{E}_{r}^{s}(P)
$$


where

$$
\mathcal{E}_{r}^{s}(P)= \begin{cases}H_{r}^{s}(P)=\left(2^{1-s}-1\right)^{-1}\left[\left[\sum_{i=1}^{n} p_{i}^{r}\right]^{\frac{s-1}{r-1}}-1\right], & r \neq 1, s \neq 1 \\ H_{1}^{S}(P)=\left(2^{1-s}-1\right)^{-1}\left[2^{(1-s) H(P)}-1\right], & r=1, s \neq 1 \\ H_{r}^{1}(P)=(1-r)^{-1} \log _{2}\left[\sum_{i=1}^{n} p_{i}^{r}\right], & r \neq 1, s=1 \\ H(P)=-\sum_{i=1}^{n} p_{i} \log _{2} p_{i}, & r=1, s=1\end{cases}
$$

\subsection{Unified $(r, s)$-Inaccuracies}

The three different kinds of unified $(r, s)$-inaccuracy measures are given by

$$
\alpha \mathcal{E}_{r}^{s}(P \| Q)= \begin{cases}{ }^{\alpha} H_{r}^{s}(P \| Q)=\left(2^{1-s}-1\right)^{-1}\left[{ }^{\alpha} K_{r}(P \| Q)^{\frac{s-1}{r-1}}-1\right], & r \neq 1, s \neq 1 \\ H_{1}^{s}(P \| Q)=\left(2^{1-s}-1\right)^{-1}\left[2^{(1-s) H(P \| Q)}-1\right], & r=1, s \neq 1 \\ { }^{\alpha} H_{r}^{1}(P \| Q)=(1-r)^{-1} \log _{2}\left[{ }^{\alpha} K_{r}(P \| Q)\right], & r \neq 1, s=1 \\ H(P \| Q)=-\sum_{i=1}^{n} p_{i} \log _{2} q_{i}, & r=1, s=1\end{cases}
$$

$\alpha=1,2$ and 3, where

$$
\begin{gathered}
{ }^{1} K_{r}(P \| Q)=\sum_{i=1}^{n} p_{i} q_{i}^{r-1}, \\
{ }^{2} K_{r}(P \| Q)=\left[\sum_{i=1}^{n} p_{i} q_{i}^{(r-1) / r}\right]^{r}, r \neq 0
\end{gathered}
$$

and.

$$
{ }^{3} K_{r}(P \| Q)=\frac{\sum_{i=1}^{n} p_{i}^{r}}{\sum_{i=1}^{n} p_{i}^{r} q_{i}^{1-r}}
$$

The above measures are obtained from the unified $(t, s)$-mean (18) in the following way:

(i) Take $v_{i}=p_{i}, w_{i}=q_{i}, \forall i=1,2, \cdots, n$ and $t=r-1$ in (18), we get $\Phi_{t}^{s}(V \| W)=$ ${ }^{1} \mathcal{E}_{r}^{s}(\mathbb{P} \| Q)$.

(ii) Take $v_{i}=p_{i}, w_{i}=q_{i}, \forall i=1,2, \cdots, n$ and $t=\frac{r-1}{r}$, in (18), we get $\Phi_{t}^{s}(V \| W)=$ ${ }^{2} \varepsilon_{r}^{s}(P \| Q)$.

(iii) Take $v_{i}=p_{i}^{r} / \sum_{k=1}^{n} p_{k}^{r}, w_{i}=q_{i}, \forall i=1,2, \cdots, n$ and $t=1-r$ in (18), we get $\Phi_{t}^{s}(V \| W)={ }^{3} \mathcal{E}_{r}^{s}(P \| Q)$.

It can easily be checked that

$$
{ }^{\alpha} \mathcal{E}_{r}^{s}(P \| Q)=\mathcal{E}_{r}^{s}(P),(\alpha=1,2 \text { and } 3)
$$


for $P=Q$ when $\alpha=1$ and 3 , and $Q=P^{r} \in \triangle_{n}^{r}$, for $\alpha=2$, where

$$
P^{r}=\left[\frac{p_{1}^{r}}{\sum_{k=1}^{n} p_{k}^{r}}, \cdots, \frac{P_{n}^{r}}{\sum_{k=1}^{n} p_{k}^{r}}\right] \in \triangle_{n}^{r}
$$

\subsection{Unified $(r, s)$-Relative Information}

Take $v_{i}=p_{i}, w_{i}=p_{i} / q_{i}, \forall i=1,2, \cdots, n$ and $t=r-1$, in (18), we get

$$
\Phi_{t}^{s}(V \| W)=-\mathcal{F}_{r}^{s}(P \| Q)
$$

where

$$
\mathcal{F}_{r}^{s}(P \| Q)= \begin{cases}D_{r}^{s}(P \| Q)=\left(1-2^{1-s}\right)^{-1}\left[\left[\sum_{i=1}^{n} p_{i}^{r} q_{i}^{1-r}\right]^{\frac{s-1}{r-1}}-1\right], & r \neq 1, s \neq 1 \\ D_{1}^{s}(P \| Q)=\left(1-2^{1-s}\right)^{-1}\left[2^{(s-1) D(P \| Q)}-1\right], & r=1, s \neq 1 \\ D_{r}^{1}(P \| Q)=(r-1)^{-1} \log _{2}\left[\sum_{i=1}^{n} p_{i}^{r} q_{i}^{1-r}\right], & r \neq 1, s=1 \\ D(P \| Q)=\sum_{i=1}^{n} P_{i} \log _{2}\left(\frac{p_{i}}{q_{i}}\right), & r=1, s=1\end{cases}
$$

We can write

$$
\mathcal{F}_{r}^{s}(P \| U)=n^{s-1}\left[\mathcal{E}_{r}^{s}(U)-\mathcal{E}_{r}^{s}(P)\right]
$$

where $U=\left(\frac{1}{n}, \cdots, \frac{1}{n}\right) \in \triangle_{n}$, and

$$
\mathcal{E}_{r}^{s}(U)= \begin{cases}\left(2^{1-s}-1\right)^{-1}\left[n^{1-s}-1\right], & s \neq 1 \\ \log _{2} n, & s=1\end{cases}
$$

we can generalize the measure (18) for two probability distributions in the following way:

$$
\bar{\Phi}_{t}^{s}\left(V_{1}, V_{2} \| W_{1}, W_{2}\right)=C E\left\{\phi_{t}^{s}\left(V_{1}, V_{2} \| W_{1}, W_{2}\right) \mid t \neq 0, s \neq 1\right\}
$$

where

$$
\phi_{t}^{s}\left(V_{1}, V_{2} \| W_{1}, W_{2}\right)=\left(2^{1-s}-1\right)^{-1}\left\{\left[\frac{1}{2} \sum_{i=1}^{n}\left[v_{1 i} w_{1 i}^{t}+v_{2 i} w_{2 i}^{t}\right]\right]^{\frac{s-1}{t}}-1\right\}, t \neq 0, s \neq 1
$$

for all $V_{1}, V_{2} \in \triangle_{n}$, and $W_{1}, W_{2} \in \Gamma_{n}$.

Similar to Section 4, some properties of the measure (31) can be extended easily.

In view of (31) and (32), we have the following unified $(r, s)$-divergence measures as particular cases. 


\subsection{Unified $(r, s)$-J-Divergences}

Take $w_{1 i}=\frac{v_{1 i}}{v_{2 i}}, w_{2 i}=\frac{v_{2 i}}{v_{1 i}}, V_{1}=P, V_{2}=Q$ and $t=r-1$ in (32) and in view of (31), we have

$$
\Phi_{t}^{s}\left(V_{1}, V_{2} \| W_{1}, W_{2}\right)=-\frac{1}{2}{ }^{1} \mathcal{L}_{r}^{s}(P \| Q),
$$

where

$$
{ }^{1} \mathcal{L}_{r}^{s}(P \| Q)=C E\left\{{ }^{1} J_{r}^{s}(P \| Q) \mid r \neq 1, s \neq 1\right\}
$$

with

$$
{ }^{1} J_{r}^{s}(P \| Q)=2\left(1-2^{1-s}\right)^{-1}\left\{\left[\sum_{i=1}^{n}\left[\frac{p_{i}^{r} q_{i}^{1-r}+p_{i}^{1-r} q_{i}^{r}}{2}\right]\right]^{\frac{s-1}{r-1}}-1\right\}, r \neq 1, s \neq 1
$$

and.

$$
{ }^{1} J_{1}^{1}(P \| Q)=J(P \| Q)=\sum_{i=1}^{n}\left(p_{i}-q_{i}\right) \log _{2}\left(\frac{p_{i}}{q_{i}}\right) .
$$

We can also write

$$
J(P \| Q)=D(P \| Q)+D(Q \| P)
$$

In (34) replace $D(P \| Q)$ by $\mathcal{F}_{r}^{s}(P \| Q)$, we get an alternative way to generalize the Jdivergence measure:

$$
{ }^{2} \mathcal{L}_{r}^{s}(P \| Q)=\mathcal{F}_{r}^{s}(P \| Q)+\mathcal{F}_{r}^{s}(Q \| P)
$$

In particular when $r=s$, we have

$$
{ }^{1} \mathcal{L}_{r}^{s}(P \| Q)={ }^{2} \mathcal{L}_{r}^{s}(P \| Q)=\mathcal{L}_{s}^{s}(P \| Q)
$$

where

$$
\mathcal{L}_{s}^{s}(P \| Q)= \begin{cases}J_{s}^{s}(P \| Q), & s \neq 1 \\ J(P \| Q), & s=1\end{cases}
$$

with

$$
J_{s}^{s}(P \| Q)=\left(1-2^{1-s}\right)^{-1}\left[\sum_{i=1}^{n}\left(p_{i}^{s} q_{i}^{1-s}+q_{i}^{s} p_{i}^{1-s}\right)-2\right], s \neq 1
$$

\subsection{Unified $(r, s)$ - R-Divergences}

Take $w_{1 i}=\frac{2 v_{1 i}}{v_{1 i}+v_{2 i}}, w_{2 i}=\frac{2 v_{2 i}}{v_{1 i}+v_{2 i}}, V_{1}=P, V_{2}=Q$ and $t=r-1$ in (32) and in view of (31), we have

$$
\Phi_{t}^{s}\left(V_{1}, V_{2} \| W_{1}, W_{2}\right)=-{ }^{1} \mathcal{K}_{r}^{s}(P \| Q) .
$$

where

$$
{ }^{1} \mathcal{K}_{r}^{s}(P \| Q)=C E\left\{{ }^{1} R_{r}^{s}(P \| Q) \mid r \neq 1, s \neq 1\right\}
$$


with

$$
{ }^{1} R_{r}^{s}(P \| Q)=\left(1-2^{1-s}\right)^{-1}\left\{\left[\sum_{i=1}^{n}\left[\frac{p_{i}^{r}+q_{i}^{r}}{2}\right]\left[\frac{p_{i}+q_{i}}{2}\right]^{1-r}\right]^{\frac{s-1}{r-1}}-1\right\}, r \neq 1, s \neq 1 .
$$

and

$$
\begin{aligned}
{ }^{1} R_{1}^{1}(P \| Q)=R(P \| Q) & =\frac{1}{2}\left[\sum_{i=1}^{n}\left(p_{i} \log p_{i}+q_{i} \log q_{i}\right)\right]-\sum_{i=1}^{n}\left(\frac{p_{i}+q_{i}}{2}\right) \log \left(\frac{p_{i}+q_{i}}{2}\right) \\
& =H\left(\frac{P+Q}{2}\right)-\frac{H(P)+H(Q)}{2}
\end{aligned}
$$

We can also write

$$
R(P \| Q)=\frac{1}{2}\left[D\left(P \| \frac{P+Q}{2}\right)+D\left(Q \| \frac{P+Q}{2}\right)\right] .
$$

Replacing $D(P \| Q)$ by $\mathcal{F}_{r}^{s}(P \| Q)$ in the expression (39), we get

$$
{ }^{2} \mathcal{K}_{r}^{s}(P \| Q)=\frac{1}{2}\left[\mathcal{F}_{r}^{s}\left(P \| \frac{P+Q}{2}\right)+\mathcal{F}_{r}^{s}\left(Q \| \frac{P+Q}{2}\right)\right]
$$

In particular for $r=s$, we get

$$
{ }^{1} \mathcal{K}_{r}^{s}(P \| Q)={ }^{2} \mathcal{K}_{r}^{s}(P \| Q)=\mathcal{K}_{s}^{s}(P \| Q),
$$

where

$$
\mathcal{K}_{s}^{s}= \begin{cases}R_{s}^{s}(P \| Q), & s \neq 1 \\ R(P \| Q), & s=1\end{cases}
$$

with

$$
R_{s}^{s}(P \| Q)=\left(1-2^{1-s}\right)^{-1}\left[\sum_{i=1}^{n}\left[\frac{p_{i}^{s}+q_{i}^{s}}{2}\right]\left[\frac{p_{i}+q_{i}}{2}\right]^{1-s}-1\right], s \neq 1
$$

Thus we see that the measures $\mathcal{E}_{r}^{s}(P),{ }^{\alpha} \mathcal{E}_{r}^{s}(P \| Q)(\alpha=1,2$ and 3$), \mathcal{F}_{r}^{s}(P \| Q)$, ${ }^{1} \mathcal{L}_{r}^{s}(P \| Q)$ and ${ }^{1} K_{r}^{s}(P \| Q)$ can be obtained as particular cases of $\Phi_{t}^{s}(V \| W)$, and the measures ${ }^{2} \mathcal{L}_{r}^{s}(P \| Q)$ and ${ }^{2} \mathcal{K}_{r}^{s}(P \| Q)$ are considered as alternative ways of generalizations A third way to generalize the R-divergence is followed by replacing $H(P)$ by $\mathcal{E}_{r}^{s}(P)$ in (38):

$$
{ }^{3} \mathcal{L}_{r}^{s}(P \| Q)=\mathcal{E}_{r}^{s}\left(\frac{P+Q}{2}\right)-\frac{\mathcal{E}_{r}^{s}(P)+\mathcal{E}_{r}^{s}(Q)}{2}
$$

Remark 2. In view of the Results 4-8, the above information theoretic measures lead us to very interesting properties. Some more properties of the measures $\mathcal{E}_{r}^{s}(P)$, 
$\alpha \mathcal{E}_{r}^{s}(P \| Q)(\alpha=1,2$ and 3$)$ and $\mathcal{F}_{r}^{s}(P \| Q)$ are recently studied by Taneja (1992). Some properties of the measures ${ }^{\alpha} \mathcal{L}_{r}^{s}(P \| Q)$ and ${ }^{\alpha} \mathcal{K}_{r}^{s}(P \| Q)(\alpha=1$ and 2) are extensively studied in Taneja (1989) Taneja et al.(1989) for $r \in(0, \infty)$ and $s \in(-\infty, \infty)$, while for $r \in(-\infty, 0)$, the measures ${ }^{\alpha} \mathcal{L}_{r}^{s}(P \| Q)$ and ${ }^{\alpha} \mathcal{K}_{r}^{s}(P \| Q)(\alpha=1$ and 2$)$ and $\mathcal{F}_{r}^{s}(P \| Q)$ become negative. Some connections with Fisher's information measure can be seen in Salicrú and Taneja (1992). Result 7 generalizes the one studied by Shiva et al. (1973), Taneja (1989) and Zografos et al. (1989). Detailed studies of the Result 7 are given in Quesada and Taneja (1992).

\section{Acknowledgements}

The authors are thankful to the reviewer for comments and suggestions on an earlier version of the paper.

\section{References}

[1] E.F. Beckenbach and R. Bellman, Inequalities, Springer-Verlag, New York, 1971.

[2] G.E.P. Box and D.R. Cox, "An Analysis of transformations," J. R. Statist. Soc. Ser. B, 26, 1964, 211-252.

[3] G.E.P. Box and G.C. Tiao, Bayesian Inference in Statistical Analysis, Addison-Wesley, Reading Mass, 1973.

[4] L.D. Broemeling, "Box and Cox Transformation," Encyclopedia of Statistical Sciences, 1, 1982, 306-307.

[5] R.G. Gallager, Information Theory and Reliable Communication, J. Wiley and Sons, 1968.

[6] G.H. Hardy, J.E. Littlewood and G. Polya, Inequalities, Cambridge, University Press, London, 1.934.

[7] F. Jelinek, Probabilistic Information Theory, McGraw Hill, New York, 1968.

[8] V. Quesada and I.J. Taneja, "Order Preserving Property of Unified $(r, s)$-Information Measures," Soochow J. Math., 18(1), 1992, 379-395.

[9] S. Shiva, N. A.hmed and N. Georganas, "Order Preserving Measures of Information," J. Appl. Probl., 10, 1973, 666-670.

[10] I.J. Taneja, "On Generalized Information Measures and their Applictions," Ad. Elect. and Electr. Physics, 76, 1989, 327-413.

[11] I.J. Taneja, On Unified $(r, s)$-Information Measures, communicated, 1992.

[12] I.J. Taneja, L. Pardo, D. Morales and M.L. Menendez, "On Generalized Information and Divergence Measures and Their Applications: A Brief Review," Questiio, 13, 1989, 47-73.

[13] M. Salicrú and I.J. Taneja, "Connections of Generalized Divergence Mesures with Fisher Information Matrix," to appear in Information sciences, 72, 1993, 251-269.

[14] K. Zografos, K. Ferentinos and T. Papaionnou, "Order Preserving Property of Measures of Information," Commun. Statist. Theory Math., 18(7), 1989, 2647-2656.

Departamento de Estadística e I.O., Facultad de Matemáticas, Universidad Complutense de Madrid, 28.040-Madrid, Spain.

Departamento de Matemática, Universidade Federal de Santa Catarina, 88.049- Florianópolis, SC, Brazil. Mailing Address : Inder Jeet Taneja, Rua Capitão Américo, 26, Corrego Grande, 88.037-060- Florianópolis, SC, BRAZIL. 